\title{
Mindfulness-Based Stress Reduction Specifically Improves Social Anhedonia among Adults with Chronic Stress
}

\author{
Corinne N. Carlton ${ }^{1}$, Ligia Antezana ${ }^{1}$, Katelyn M. Garcia ${ }^{1}$, Holly Sullivan-Toole ${ }^{1,2}$ \\ \& John A., Richey ${ }^{1}$
}

\footnotetext{
${ }^{1}$ Virginia Tech, Department of Psychology, 109 Williams Hall, Blacksburg, VA, 24061

${ }^{2}$ Temple University, Department of Psychology, 1701 North 13th Street, Philadelphia, PA, 191221
}

\author{
Correspondence concerning this paper should be addressed to Corinne Carlton, Virginia Tech, \\ Department of Psychology, 109 Williams Hall, Blacksburg, VA, 24061. Email: \\ corinnecarlton@vt.edu.
}

Keywords: mindfulness; mindfulness-based stress reduction; anhedonia; stress; social anhedonia 


\begin{abstract}
This randomized controlled trial examined the effects of mindfulness on anhedonic symptoms in a sample of adults reporting high levels of chronic stress. Meditation-naïve adults ( $\mathrm{N}=68$, Mage $=32,62 \%$ female) were randomized to either an 8-week MBSR intervention group $(\mathrm{N}=35)$, or to a waitlist control group $(\mathrm{N}=33)$. We hypothesized that changes in mindfulness would mediate the relationship between condition and changes in anhedonic symptoms. Additionally, the present study aimed to determine if other theoretically-linked mechanisms (i.e., stress, negative affect(NA), depression) were involved in producing changes in anhedonic symptoms. Results provided evidence for full mediation of the effect of MBSR on social anhedonia through its essential mechanism of $\Delta$ Mindfulness. These results highlight specificity of anhedonic symptoms targeted by MBSR, with social anhedonia symptoms being modified by changes in mindfulness whereas other anhedonic domains were not. Additionally, although associative relationships were present for stress, depression, NA, and anhedonic symptoms, no mediational relationships emerged.
\end{abstract}

Keywords: mindfulness; mindfulness-based stress reduction; anhedonia; stress; social anhedonia 


\section{Mindfulness-Based Stress Reduction Improves Social Anhedonia among Adults with}

\section{Chronic Stress}

Anhedonia refers to diminished interest or pleasure in response to stimuli that were previously perceived as rewarding (American Psychiatric Association, 2013), as well as decreased motivation and reactivity to stimuli that were formerly construed as pleasurable (e.g., Slattery, Markou, \& Cryan, 2007). Extensive prior work has linked anhedonia to both the development and exacerbation of a variety of negative health outcomes including both physical (e.g., cardiovascular disease, inflammatory diseases) and mental illnesses (e.g., depression, anxiety; Lupien, Juster, Raymond, \& Marin, 2018; Pizzagalli, 2014). Despite its robust association with negative sequelae, the mechanisms of anhedonia development and maintenance remain incompletely understood. Current front-line interventions for depression and other conditions involving anhedonia including adaptations of cognitive behavioral therapy and related approaches do not explicitly target rewardbased motivational systems. Yet dysfunction within this broadly defined neurobiological system has been previously linked to the development of anhedonia (e.g., Pizzagalli, Iosifescu, Hallett, Ratner, \& Fava, 2008; Zhou, Nie, Wang, Wang, \& Zheng, 2019; for reviews see Der-Avakian \& Markou, 2012; Höflich, Michenthaler, Kasper, \& Lanzenberger, 2019). Moreover, front-line pharmacotherapies (e.g. SSRIs) also do not target reward-based deficits in anhedonia (Dunlop \& Nemeroff, 2007; McCabe, Cowen, \& Harmer, 2009; Nutt et al., 2007). Perhaps as a consequence, anhedonic symptoms tend to persist even after the completion of both psychological and pharmacological treatment across various forms of psychopathology (e.g., Craske, Meuret, Ritz, Treanor, \& Dour, 2016; Lambert et al., 2018; McCabe, Mishor, Cowen, \& Harmer, 2010; McClintock et al., 2011; Wolf, 2006). Further, anhedonia is a predictor of suboptimal treatment outcomes (Morris, Bylsma, \& Rottenberg, 2009; Spijker, Bijl, De Graaf, \& Nolen, 2001; Vrieze 
et al., 2014), suggesting that targeting anhedonia in the context of treatment may catalyze or complement existing forms of intervention efforts. To this end, elucidating key mechanisms underlying the reduction of anhedonia is crucial for developing adjunctive therapies that can successfully target anhedonia symptoms. Accordingly, the purpose of the work outlined here is to probe the effects of mindfulness training on anhedonic symptoms, and to further test the hypothesis that observed changes in anhedonia symptomatology are due to improvements in mindfulness as a presumed mechanism of action.

Prior theoretical work suggests that mindfulness training and perhaps Mindfulness-Based Stress Reduction (MBSR) in particular may modulate anhedonia by re-establishing connections between reward-predicting stimuli and their potential for pleasurable consequences (e.g., Richey et al., 2019). Mindfulness can be broadly defined as a process of cultivating non-judgmental awareness of the present moment, including purposeful attention to present thoughts, feelings and sensations (Kabat- Zinn, 1990). Mindfulness-based interventions (MBIs) including MBSR (KabatZinn, 2003) and Mindfulness-Based Cognitive Therapy (MBCT; Segal, Williams, \& Teasdale, 2018) have been previously examined as potential methods for directly engaging depressive symptomatology (Chi, Bo, Liu, Zhang, \& Chi, 2018; Kenny \& Williams, 2007; Serpa, Taylor, \& Tillisch, 2014). MBSR is perhaps the most intensively studied therapeutic adaptation of mindfulness training, and has further been established as an efficacious treatment for reducing symptoms of depression, anxiety and other disorders that include an anhedonic component (Chi et al., 2018; Khoury, Sharma, Rush, \& Fournier, 2015; Li \& Bressington, 2019). Although the mechanisms by which these symptoms are modulated by MBIs remain an active area of inquiry, it has been previously theorized that MBIs may improve anhedonic symptoms by targeting rewardbased motivational systems (Richey et al., 2019). Two relevant premises within MBSR include: 
(1) identification and/or noticing the occurrence of thoughts as they arise; and (2) nonjudgmentally registering negative thoughts as mental events rather than objective representations of reality. Although these specific components within MBSR are not necessarily thought to lead to fewer ruminative or negative thoughts, they instead may reduce the impact of negative emotions once activated (Brewer, Van Dam, \& Davis, 2015). Moreover, MBSR specifically may hold promise as a treatment approach for reducing anhedonic symptoms by reducing the impact of stress (for meta-analysis see Khoury et al., 2015), which is a well-established precursor to the development of anhedonia (e.g., Pizzagalli, 2014; Stanton, Holmes, Chang, \& Joormann, 2019; for review Carlton, Sullivan-Toole, Ghane, \& Richey, 2020a).

Chronic stress is perhaps the best psychological predictor of anhedonia. Recent reviews (e.g., Höflich et al., 2019; Pizzagalli, 2014; Stanton et al., 2019) theorize that anhedonia itself is the result of dysfunctional stress-reward interactions. Moreover, various studies spanning both the preclinical literature and human studies have provided evidence for this theoretical relationship between stress and anhedonia (e.g., Berenbaum \& Connelly, 1993; Bogdan \& Pizzagalli, 2006; Rygula et al., 2005). One of the earliest demonstrations of this linkage in human studies was provided by Berenbaum and Connelly (1993), where it was determined that both U.S. Army cadets and college students experienced diminished ability to experience pleasure after experiencing stressful events. Bogdan and Pizzagalli (2006) later probed the specific linkage between stress and anhedonia by demonstrating that individuals reporting greater anhedonic symptoms suffered more substantial hedonic deficits under conditions of acute stress. Of note, these relationships have not been assessed within anhedonic samples or within samples characterized by chronic stress. Although in a recent epidemiologic study Dodell-Feder and Germine (2018) determined that individuals living in high stress situations (e.g., social and economic disadvantages) demonstrated 
high levels of social anhedonia; suggesting a potential vulnerability for the stress-anhedonia relationship within social domains. Given that anhedonia has long been recognized as a vulnerability marker for subsequent affective pathology (e.g., Lupien et al., 2018), premorbid anhedonic samples may therefore be especially informative in determining mechanisms involved in the development and maintenance of anhedonic symptoms, separate from depressive symptomatology. Moreover, the characterization of chronic stress-anhedonia relationships is particularly important given that in diagnostic cases, such as major depressive disorder severe anhedonia may have already become entrenched or so well learned that it may be more difficult to reverse, which could also explain the consistent reports of the treatment refractory nature of anhedonia in clinical samples (Craske et al., 2016; Lambert et al., 2018; McCabe et al., 2010; McClintock et al., 2011). For example, in a study by McMakin and colleagues (2012), the presence of anhedonia in patients with major depressive disorder was the only dimension that predicted longer remission times, suggesting a unique tie between anhedonia and diagnostic severity. Therefore, it is critical to investigate individuals reporting chronic stress as they are a group at high risk for developing anhedonia and as such offer an opportunity to probe the pathogenesis of anhedonic symptoms.

In terms of potential mechanisms of action, connections between mindfulness training and systems of reinforcement-based learning may be at the core of MBSR's potential efficacy to reduce symptoms of anhedonia. Richey and colleagues (2019) theorized that the pre-anhedonic period is characterized by repeated experiences with frustrative non-reward, where energy expenditure does not produce the intended or desired outcome. Repeated experiences of this nature may eventually disconnect formerly reward-predicting cues in the environment from a linked expectation for pleasurable outcomes, as the individual learns that pursuit of such cues is unlikely to produce any 
useful result. Thus, energy is conserved upon future encounters with such environmental cues, consistent with general symptomatology clustered within the definition of anhedonia (i.e. reductions in motivation, reduced experiences of pleasure). Mindfulness training may be unique in its potential to target these reinforcement-based learning mechanisms involved in the development of anhedonia by re-establishing the broken association between pleasurable experiences and the cues that predict them. For example, intentionally cultivating mindful awareness of bodily sensations during experiences such as eating, social interaction or physical exercise may promote rediscovery of the environmental cues that naturally precede them. Whereas during the anhedonic period such cues may typically fail to trigger motivated action, motivational tone may be reinstated by mindfulness practice through repeated explicit pairings between the physical experience of pleasure and the activities that precede it, a process which is reified through formal meditation practice as implemented in MBSR. A central contribution of this theoretical work is the prediction that mindfulness treatment should enhance detection, registration, and processing of cues that predict reward and therefore increase motivation to pursue their rewarding consequences.

Recent work has demonstrated that anhedonia comprises a diverse spectrum of reward difficulties, which includes deficits in interests and desire, anticipation, motivation, effort, and consummatory pleasure (Rizvi, Lambert, \& Kennedy, 2018; Rizvi et al., 2015). Yet, most anhedonia measures have historically conceptualized anhedonia as a uni-dimensional construct typically related to derivation of pleasure from environmental stimuli (Ribot, 1896). The Dimensional Anhedonia Rating Scale (DARS; Rizvi et al., 2015) is one of the first measures to examine the dimensionality inherent within anhedonia, with attention to several domains of rewarding stimuli, including pleasure from social interactions, hobbies and pastimes, food and 
drinks, and sensory experiences. The DARS includes domain scores for each of these anhedoniarelevant domains. Insofar as anhedonia is a transdiagnostic symptom found across multiple psychiatric disorders, examining the dimensionality of anhedonia symptoms is an important step in understanding how anhedonia may function in the pathogenesis and maintenance of affective pathology. The examination of anhedonic dimensionality is of particular relevance for the current study, given that theoretical work by Richey and colleagues (2019) emphasizes the potential for MBSR to engage and specifically recouple social sources of reward with their earliest consistent predictors. However, despite the inherent promise of MBSR as an effective treatment approach for reducing anhedonic symptoms, potentially through this mechanism, no studies to our knowledge have specifically evaluated the impact of MBSR across dimensions of anhedonia.

In light of prior work suggesting that MBSR is an efficacious treatment for reducing symptoms that comprise an anhedonic component (Chi et al., 2018; Khoury et al., 2015; Li \& Bressington, 2019), as well as our theoretical model predicting that MBSR may improve symptoms of social anhedonia by targeting reward-based motivational systems (Richey et al., 2019), we hypothesized that random assignment to MBSR would modulate anhedonia symptoms, and social anhedonia symptoms more specifically, albeit indirectly via its presumed core mechanism of improvements in trait mindfulness. Therefore, the present study sought out to accomplish the following aims. First, to identify the effect of MBSR on change in domain-specific anhedonic symptoms. Second, to determine if improvement in mindfulness specifically mediates the relationship between random assignment to MBSR versus waitlist (WL) control and improvement in anhedonic symptoms. We specifically hypothesized that (H1) MBSR would result in reduced anhedonic symptoms across all domains, but have a stronger effect on social anhedonic symptoms and that (H2) improvements in mindfulness would act as a mediator in the relationship between 
condition and anhedonic symptom improvement. Additionally, in light of previous literature indicating that MBSR results in decreased self-reported measures of stress, depression, and negative affect (NA) and their theoretical linkages to anhedonia (e.g., Chi et al., 2018; Cook, Lanza, Chu, Baker, \& Piper, 2017; Pelizza \& Ferrari, 2009; Snippe, Dziak, Lanza, Nyklíček, \& Wichers, 2017), the present study also aimed to probe the specificity of the presumed mediational status of mindfulness change, by establishing whether other theoretically-linked mechanisms (i.e., stress, NA, depression) were similarly involved in producing any observed changes in anhedonic symptoms. Therefore, the current study further aimed to increase specificity by evaluating the relationship between treatment with MBSR with stress, depression, NA, and anhedonic symptoms and to determine if improvements in stress, depression, or NA act as mediators for the relationship between MBSR and improvement in anhedonic symptoms. We specifically hypothesized that (H3) these negatively-valenced measures would be correlated with anhedonia change in the context of the RCT; however, we predicted that $(\mathrm{H} 4)$ they would not mediate the relationship between MBSR and changes in anhedonia.

\section{Method}

\section{Participants}

All participants provided written informed consent as approved by the Virginia Tech institutional review board (IRB). Participants were recruited via online advertisements as well as flyers posted throughout the community. Advertisements introduced the study as a potential treatment for improving chronic stress. Interested individuals completed a brief phone screening during which their medication history was assessed for potential eligibility, and participants were informed of the nature of the treatment and study. Exclusionary criteria for the present study included current medical ailments (e.g., diabetes, psoriasis, or other inflammatory skin condition, 
peripheral vascular disease, peripheral arterial disease, Raynaud's disease, or any other diagnosed circulatory disorders, involuntary motor disorders). In addition, since the larger study procedures included blood draws, being allergic to adhesive tape or a history of problems with blood draws were also grounds for exclusion. Individuals were also excluded from participating if they reported a history of alcohol or non-prescription drug abuse or were currently taking any of the following medications: inhaled steroids or any corticosteroids, any medication that affects central nervous system function, or psychotropic medications. Additionally, individuals who reported a phobia of needles, or those who were currently enrolled in another form of therapy were excluded from participating. Finally, individuals were excluded if they worked a night shift or if they met criteria for two of the following concurrently: hypertension, hyperlipidemia, high cholesterol, smoked cigarettes, or had a family history of coronary or atherosclerotic disease. The CONSORT flow diagram for this study is presented in Figure 1.

Participants were deemed eligible if they were between the ages of 18 and 55, had no previous experience with meditation, no daily practice with other mind-body techniques (e.g., yoga, tai-chi, qi gong), and were in good general physical health. The average age of all participants was approximately 32 years $(S D=11.5)$, and the sample was predominantly female (62.3\%). Approximately $71 \%$ of participants identified as White/Caucasian, $4 \%$ as Black/African American, $13 \%$ as Asian, and $12 \%$ as Other; additionally, $6 \%$ of all participants identified as being of Hispanic/Latinx ethnicity. With regard to socioeconomic status (SES), level of education, employment status, and income information were collected. Approximately $1.8 \%$ of our sample reported finishing grade school, 5.3\% reported finishing high school or the equivalent (e.g., GED), $14.0 \%$ reported completing some college, $38.6 \%$ reported earning a college degree, and $40.3 \%$ reported earning an advanced degree. Our sample also reported on their employment status, with 
$22.8 \%$ indicating that they were not currently employed, $38.6 \%$ reporting that they were full-time employees, and $36.8 \%$ reporting that they were part-time employees, with approximately $1.8 \%$ of participants opting to not respond. With regard to income, $22.8 \%$ of our sample reported earning between $\$ 0-10,000,19.3 \%$ reported earning between $\$ 10,001-20,000,14.0 \%$ reported earning between $\$ 20,001-40,000,19.3 \%$ reported earning between $\$ 40,001-65,000,10.5 \%$ reported earning between $\$ 65,001-100,000$, and $10.5 \%$ reported earning more than $\$ 100,000$ with $3.6 \%$ of participants opting to not respond.

\section{Assessment Schedule}

Enrolled participants were randomized to either an 8-week MBSR group intervention $(\mathrm{N}=$ 35) or waitlist (WL) control $(\mathrm{N}=33)$ based on a random number generator. All participants completed baseline assessment in person including self-report measures prior to random assignment to condition. Participants in the MBSR group began to participate in the treatment approximately 2-weeks subsequent to baseline assessment, on average. At 4 weeks subsequent to the first session of the MBSR intervention, participants in both groups filled out midpoint selfreport measures. Following completion of midpoint measures, participants in the MBSR group continued treatment. Upon completion of the full MBSR course (8-weeks subsequent to session 1), participants in both conditions were asked to fill out endpoint self-report measures.

\section{Mindfulness-Based Stress Reduction (MBSR) protocol}

MBSR is a widely available program with more than three meta-analyses (Grossman, Niemann, Schmidt, \& Walach, 2004; Hofmann, Sawyer, Witt, \& Oh, 2010; Khoury et al., 2015) supporting its efficacy across a range of conditions including chronic stress. MBSR is administered in-person across 8 once-weekly sessions in a group format. Exercises are drawn mainly from the Vipassana tradition including breath awareness, guided body awareness, walking meditation and 
gentle yoga. In the current study, we followed the most recent available MBSR curriculum (Santorelli, Kabat-Zinn, Blacker, Meleo-Meyer, \& Koerbel, 2017). Groups met for 2.5 hours weekly and in addition, participants engaged in approximately 30-45 minutes of daily home meditation practice. The MBSR program was carried out by a doctoral level clinician who earned “qualified" status from the University of Massachusetts - the founding site for MBSR

\section{Waitlist (WL) protocol}

Participants randomly assigned to the WL condition followed the same assessment schedule as the MBSR group (baseline, 4-week, and 8-week measurements), but received no intervention and were furthermore asked to wait approximately 12 weeks to enroll in our next available MBSR group, if desired. The decision to use a WL format followed from ethical guidelines which suggest that permanently withholding an intervention that is theorized to be efficacious may act to increase risk among individuals randomly assigned in the comparison condition, who presumptively sought participation in an effort to reduce the negative consequences of chronic stress. Thus, we opted to allow WL participants to enroll in MBSR subsequent to the completion of their involvement in the WL condition. Also for ethical reasons, those assigned to WL were not explicitly instructed to refrain from any particular activities such as yoga or other forms of mindfulness training; however, these were re-assessed at follow-up to ensure that WL participants did not engage in training of this nature. Study policies required that any participant who enrolled in new mindfulness training including MBSR or yoga instruction would be disenrolled from data collection (but still allowed to enroll in the next available MBSR group). However, results indicated that no WL participants were excluded from data collection on this basis. Note also that data reported here for the MBSR group do not include any subject who was formerly enrolled in the WL condition. 
*******Insert Figure 1 About Here******

\section{Behavioral Measures}

Five Facet Mindfulness Questionnaire (FFMQ; Baer, Smith, Hopkins, Krietemeyer, \& Toney, 2006). The FFMQ is a 39 -item questionnaire that measures five different domains of mindfulness, including observing, describing, acting with awareness, nonjudgement of inner experience, and non-reactivity to inner experience. Participants were asked to rate statements regarding these domains on a 5-point Likert-type scale, ranging from 1 ("never or very rarely true") to 5 ("very often or always true"). Higher scores indicate greater trait mindfulness. This scale has demonstrated strong reliability (Baer et al., 2008), and had an excellent internal consistency within this study; Cronbach's alphas were 0.92 for baseline and 0.93 for endpoint. The FFMQ was used as a mediator in the proposed analyses.

Dimensional Anhedonia Rating Scale (DARS; Rizvi et al., 2015). The DARS is a 17item questionnaire that assesses anhedonia severity across 4 domains: hobbies/past-times, food/drinks, social activities, and sensory experiences. Within each domain, participants were asked to write at least two examples of things that they find rewarding/pleasurable, and were then asked to answer questions about desire, motivation, effort and pleasure for the examples that they had chosen on 5-point Likert-type scale ranging from 0 ("not at all") to 4 ("very much"). Higher scores indicate increased desire, motivation, effort, and pleasure (i.e., less anhedonia). In the current study, the measure's internal consistency was excellent; the Cronbach's alphas were 0.86 for baseline and 0.91 for endpoint. DARS total and domain scores were used as dependent variables in mediational analyses. 
Perceived Stress Scale (PSS-10; Cohen \& Williamson, 1988). The PSS-10 is a brief, 10item questionnaire aimed at measuring the degree to which participants appraise situations in their lives to be stressful over the past month. Participants were asked to select how often they felt a certain way (e.g., "In the last month, how often have you felt that things were going your way" on a 5-point Likert-type scale ranging from 0 ("never") to 4 ("very often"). The PSS-10 has been demonstrated to have good psychometric properties (Lee, 2012). Similarly, in the present study the PSS-10 also demonstrated excellent internal consistency (baseline $a=.87$, endpoint $a=.90$ ). The PSS-10 was used as a dependent variable in mediational analyses.

Positive and Negative Affect Schedule (PANAS-X; Watson \& Clark, 1999). The PANAS-X is a 60 -item measure that assess negative and positive affect. Participants were asked to indicate how often they "generally" experience listed affectivity terms on a 5-point Likert-type scale during the past few weeks. Response items ranged from 1 ("very slightly or not at all") to 5 (“extremely"). In prior work, the PANAS-X has been demonstrated to have excellent internal consistency ( $a=$ ranging from .83 to $.90 ;$ Watson \& Clark, 1999). In the current sample, alpha estimates for both scales of PANAS-X were determined to be excellent (Positive Affectivity (PA) baseline $a=.91$, endpoint $a=.92$; Negative Affectivity (NA) baseline $a=.87$, endpoint $a=.90$ ). The NA scale of the PANAS was used as a dependent variable in mediational analyses.

Beck Depression Inventory (BDI-II; Beck, Steer, \& Brown, 1996). The BDI-II is a 21question assessment of the severity of depressive symptoms over the past two weeks. Specifically, participants were asked to endorse how they felt with regard to different depression symptom dimensions on a 4-point Likert-type scale ranging from 0 to 3, with higher scores indicating greater severity of depressive symptoms. The BDI-II has been well established as a reliable and valid measure, with excellent internal consistency (Wang \& Gorenstein, 2013). Within the present study, 
the BDI-II also showed excellent internal consistency (baseline $a=.91$, endpoint $a=.93$ ). The BDI-II was used as a dependent variable in mediational analyses.

\section{Data Analysis Plan}

In order to assess associative relationships between treatment with MBSR, anhedonic symptoms, and other symptoms (i.e., depression, stress, NA) change scores were calculated (post minus pre) and we first examined zero-order Pearson correlations among baseline-endpoint change scores of all theoretically relevant variables. Second, to determine if condition would result in reduced anhedonic symptoms across all domains, but have a stronger effect on social anhedonic symptoms, linear regressions were run to predict all change scores in DARS scores at endpoint. Next, to determine if improvement in mindfulness at endpoint acts as a mediator in the relationship between condition and changes in anhedonic symptoms, mediation analyses were conducted in SPSS PROCESS macro (Hayes, 2012; model 4). Additionally, we explored dosage effects by separately probing the mediational status of mindfulness change at 4-weeks (midpoint). These steps were repeated to assess the potential mediators of depression, stress, and NA, as measured by the BDI-II, PSS-10, and PANAS-X, respectively. Mediation models were carried out using these variables if linear regressions were significant.

\section{Results}

\section{Observed Means and Correlational Relationships}

Means and standard deviations of all measures at baseline, midpoint, and endpoint for MBSR and WL are displayed in Table 1. Correlational results indicated the following associations between variables as presented in Table 2. Significant moderate correlational values emerged between condition and $\Delta$ Mindfulness $(r=.37, p<.01)$, as well as condition and $\Delta$ DARS Social 
Activities $(r=.35, p<.01)$. Significant moderate correlational associations were also demonstrated between $\Delta$ Mindfulness and $\triangle$ DARS Total $(r=.39, p<.01), \Delta$ DARS Social Activities $(r=.43, p$ $<.01)$, and $\triangle$ DARS Sensory Experiences $(r=.30, p<.05)$.

Additionally, moderate-to-strong significant relationships emerged between $\triangle \mathrm{PSS}-10$ and $\Delta$ Mindfulness $(r=.65, p<.01), \Delta$ DARS Total $(r=.35, p<.01), \Delta$ DARS Food/Drink $(r=.41, p<$ $.01)$, and $\triangle$ DARS Sensory Experiences $(r=.41, p<.01) . \Delta$ BDI-II demonstrated significant negative associations ( $r$ 's ranged between -.28 and -.48 , all $p$ 's $<.05$ ) with all study variables except $\triangle$ DARS Hobbies/Pastimes $(r=-.10, p=.48)$ and $\triangle$ DARS Sensory Experiences $(r=-.18, p$ $=.19)$. Lastly, $\Delta$ NA showed significant negative relationships with $\Delta$ Mindfulness $(r=-.45, p<$ $.01), \triangle$ DARS Total, $\triangle$ DARS Food/Drink $(r=-.32, p<.05), \Delta$ PSS-10 $(r=-.44, p<.01)$, and a significant positive relationship with $\Delta$ BDI-II $(r=.42, p<.01)$.

$* * * * *$ Insert Table 1 About Here $* * * * *$

\section{Linear Regression Analyses}

Linear regression analyses were conducted in order to determine if condition was linked to changes in anhedonic symptoms across all sub-domains of the DARS, and furthermore whether random assignment to MBSR produced a more profound effect on change in social anhedonic symptoms. Results of these analyses are displayed in Table 3, and indicate that, consistent with initial hypothesis (H1), condition was a significant predictor of $\triangle$ DARS Social Activities $(p<.01)$ only. Condition did not emerge as a significant predictor of $\triangle$ DARS Total $(p=.53), \triangle \mathrm{DARS}$ Food/Drink $(p=.48), \Delta$ DARS Sensory Experiences $(p=.53)$, or $\Delta$ DARS Hobbies/Pastimes anhedonic symptoms $(p=.95)$. In order to further increase specificity, we evaluated the 
relationship between condition and stress, depression, NA, and anhedonic symptoms. Linear regression analyses were also conducted using these variables as predictors (see Table 3). Results indicated that condition emerged as a significant predictor of $\Delta \mathrm{BDI}-\mathrm{II}(p=.01)$, but not a significant predictor of $\triangle \mathrm{PSS}-10$ nor $\triangle \mathrm{NA}$.

\section{Mediational Analysis}

To determine if improvement in mindfulness acts as a mediator for the relationship between condition and changes in anhedonic symptoms, mediational analyses were conducted in the SPSS PROCESS macro (Hayes, 2012; model 4). Since linear regression analyses revealed that, in-line with initial predictions, condition was a significant predictor of $\triangle \mathrm{DARS}$ Social Activities $(p<.01)$ at endpoint, this was the only anhedonia variable assessed in subsequent mediation models. Results from the primary mediational analysis demonstrated that the total effect of the model was significant $(p<.01)$ and that the direct effect was no longer significant after accounting for the indirect effect $(\beta=1.33, p=.10)$, suggesting full, not partial (or no), mediation (see Figure 2).

\footnotetext{
******Insert Figure 2 About Here*******
}

In addition to the primary assessment of $\Delta$ Mindfulness from baseline to endpoint, dosage effects were investigated by running the same mediational analysis for the change in mindfulness from baseline to midpoint as a mediator in the relationship between condition and $\triangle \mathrm{DARS}$ Social Activities at midpoint. Results revealed no evidence of mediation for this relationship as the direct effect was still significant $(\beta=1.76, p<.05)$ after accounting for the indirect effect (see Figure S1 in the Supplemental Material available online). 
Additionally, since linear regression results revealed condition to be a significant predictor of baseline-endpoint $\Delta$ BDI-II $(p=.01)$, and baseline-endpoint $\Delta$ DARS Social Activities $(p<.01)$, and that $\Delta \mathrm{BDI}$-II was a significant predictor of $\triangle \mathrm{DARS}$ Social Activities $(p<.05)$, these variables were entered into an additional mediational analysis through the SPSS PROCESS macro (Hayes, 2012; model 4). This additional analysis was carried out in order to determine if improvement in depression acted as a mediator for the relationship between condition and changes in anhedonic symptoms. Results from the mediational analysis demonstrated that the total effect of the model was significant $(p<.01)$ and that the direct effect demonstrated a trend significance after accounting for the indirect effect $(\beta=1.78, p=.05)$; however, the indirect effect confidence interval contained zero $($ BootLLCI $=-.42$, BootULCI $=1.31)$; providing limited evidence of mediation by baseline-endpoint changes in depression (see Figure 3). The dosage effect for baseline-midpoint (4 weeks) was not examined for depression as the mediational model was not significant at the full dose of baseline-endpoint (8-weeks).

\footnotetext{
****** Insert Figure 3 About Here*******
}

\section{Discussion}

Regarding the current study's primary aims, we specifically hypothesized that (H1) MBSR would result in reduced anhedonic symptoms across all domains but have a stronger effect on social anhedonic symptoms, and (H2) also that improvements in mindfulness would act as a mediator in the relationship between condition and anhedonic symptom improvement. These hypotheses were motivated by previous work demonstrating MBSR as an efficacious treatment for reducing symptoms of disorders that comprise an anhedonic component (Chi et al., 2018; Khoury 
et al., 2015; Li \& Bressington, 2019), as well as recent epidemiologic work on social anhedonia (Dodell-Feder \& Germine, 2018). Of note, consistent with previous literature linking stress to anhedonia (Dodell-Feder \& Germine, 2018), participants in our sample were highly anhedonic (baseline DARS Total $M=48.81$, DARS Social $M=10.85$ ), which was comparable to samples in depression (DARS Total $M=51.20$, DARS Social Activities $M=10.00$; Rizvi et al., 2015). Results indicated that treatment with MBSR did indeed reduce anhedonic symptoms; however, this was limited to the social activities anhedonic domain only, providing partial support for $\mathrm{H} 1$. One potential reason for this finding may owe perhaps to the group format of MBSR; potentially allowing improvements in mindfulness through MBSR to recouple reward experiences to social interactions more proximally than other anhedonic domains. Results also demonstrated that improvements in mindfulness fully mediated the relationship between condition and changes in social anhedonic symptoms, thus lending support to $\mathrm{H} 2$ with regard to the social anhedonia domain only. Further, results revealed a relevant dosage effect, such that improvements in mindfulness mediated the relationship between condition and improved social anhedonia at 8-weeks, but not sooner at 4-weeks; suggesting the need for the full course of MBSR to produce the observed effects.

The unique impact of MBSR on social anhedonia in our results indicates that the effect of mindfulness training on anhedonic symptoms may be more specific than previously thought. Social anhedonia, or the reduced desire for social affiliation or pleasure from interpersonal interactions, is commonly noted across several disorders (i.e., social anxiety disorder, autism spectrum disorder, depression, schizophrenia; Lambert et al., 2018) and tends to persist even after treatment (e.g., Craske et al., 2016; Lambert et al., 2018; McCabe et al., 2010; McClintock et al., 2011; Wolf, 2006). This resistance to prominent intervention approaches may be at least in part 
due to missed targeting of crucial mechanisms involved in the development and maintenance of anhedonia, which have been theorized to be rooted in reward systems (e.g., Pizzagalli et al., 2008; Zhou et al., 2019) and may be directly engaged by MBSR (e.g., Richey et al., 2019). Therefore, the central contributions of the present study are (1) that MBSR specifically improves social anhedonia symptoms; (2) improvements in mindfulness, facilitates improvements in social anhedonia symptoms. These results suggest that MBSR may indeed target motivational systems, perhaps through improvements in trait mindfulness that may recouple social situations with the experience of reward, resulting in reduced social anhedonia. The present results suggest that current treatments for disorders with a significant social anhedonic component may better target these specific symptoms with the addition of mindfulness elements or an MBSR framework.

In light of extensive prior work that has established decreases in stress, depression, and NA following MBSR, and their association with anhedonia (e.g., Chi et al., 2018; Cook et al., 2017; Pelizza \& Ferrari, 2009; Snippe et al., 2017), the present study also aimed to determine if these theoretically-linked mechanisms are involved in producing changes in anhedonic symptoms. The current study recruited a high stress sample (baseline PSS-10 $M=21.81$ ), which was above general population means (ages 18-29, PSS-10 $M=14.20$, ages 30-44 PSS-10 $M=13.00$; Cohen, 1988) and consistent with MBI samples at baseline (PSS-10 $M=19.00$; Fortney, Luchterhand, Zakletskaia, Zgierska, \& Rakel, 2013). Moreover, the current study averages in NA (baseline NA $M=22.84$ ) and depression (baseline BDI-II $M=14.95$ ) were slightly lower than clinical samples (NA $M=25.74$ BDI-II $M=19.33$; Naragon-Gainey, Watson, \& Markon, 2009). Linear regression analyses revealed that MBSR condition was only a significant predictor of changes in depression, but neither stress nor negative affect. Further, mediational analyses indicated that there was no mediational effect of changes in depression on the relationship between MBSR condition and 
social anhedonia. These results are consistent with past studies examining the impact of MBSR on improvements in depression (e.g., Baer, Carmody, \& Hunsinger, 2012); however, they do not align with the notion the MBSR produces improvements in NA and stress as seen in other studies (e.g., Gawrysiak et al., 2018; Snippe et al., 2017); which have been theoretically linked to increased anhedonic symptoms. These results are important to consider, such that they may suggest that stress might trigger anhedonia, but reducing stress may not necessarily alleviate anhedonia; however, increasing mindfulness can. Moreover, these results support previous theoretical linkages between depression and anhedonic symptoms (e.g., Pizzagalli, 2014), even with no evidence of a mediational effect being present. Altogether, this pattern of results suggests that while improving symptoms of depression may have a direct effect for decreasing social anhedonia symptoms, this may not be due specifically to the effects of MBSR, but to depressive symptomatology more broadly.

The specificity of changes in social anhedonia as a response to MBSR is an important finding that should be probed further with other MBIs, especially in the context of social anxiety disorder, autism spectrum disorder, depression, and schizophrenia (Lambert et al., 2018). More specifically, recent work using mindfulness techniques has found promise for targeting emotion regulation and social skills difficulties in autistic populations (Conner \& White, 2018; Conner et al., 2019), which is consistent with previous work demonstrating that mindfulness-based techniques decrease emotional reactivity toward social stress (Britton, Shahar, Szepsenwol, \& Jacobs, 2012). Considering the motivational/reward differences in autism and their relationship to social difficulties in this disorder (i.e., Clements et al., 2018; Dichter et al., 2012; Dichter, 2018; Kohls, Antezana, Mosner, Schultz, \& Yerys, 2018; Tschida \& Yerys, 2020), changes in mindfulness may be a catalyst for changes in neural mechanisms of social reward that warrants 
further examination. Moreover, given that the present study recruited an adult chronic stress sample, future work should determine the utility of this approach for other samples, such as adolescents. Of note, recent theoretical work has proposed that MBIs, and MBSR specifically, may hold particular relevance in engaging motivational deficits in adolescents with social anxiety disorder (Carlton, Sullivan-Toole, Strege, Ollendick, \& Richey, 2020b). Therefore, more work is needed to establish MBSR's effectiveness in younger populations in an attempt to prevent the development of more chronic anhedonic endophenotypes.

As with any study, results presented here should be evaluated in light of study limitations. For example, the present study only included self-report measures. As such, the addition of behavioral (e.g., signal-detection methodology; Pizzagalli, Jahn, \& O’Shea, 2005) or biological (e.g., hemodynamic; Keedwell, Andrew, Williams, Brammer, \& Phillips, 2005) measures of reward responsivity may add more specificity for treatment targets proposed herein. As there is a growing literature on neural mechanisms of MBIs and associations with anhedonia (e.g., Cernasov et al., 2019), it will also be important to understand changes in dimensionality and types of anhedonia to better uncover modifiable treatment mechanisms. Thus, considering the complexity of anhedonia, evaluating and disentangling specific reward pathways (i.e., interest/desire, effort, motivation, consummatory pleasure) may aid in our understanding of mechanisms by which MBIs specifically impact social anhedonia (Rizvi et al., 2018; Rizvi et al., 2015). Moreover, understanding anhedonic profiles (i.e., social, food, hobbies/pastimes, sensory) may help in our understanding of MBI treatment responsivity. Additionally, while the DARS is a valuable tool for evaluating more personalized forms of anhedonia through various subdomains, and has been established as a reliable and valid measure of anhedonia (Rizvi et al., 2015); the use of additional specific social anhedonia measures may have the potential to more deeply assess change. One such 
measure that may reveal even more information regarding social hedonic tone is the Social Anhedonia Scale (Chapman, Chapman, \& Raulin, 1976); therefore, future work should incorporate this measure. Finally, an additional limitation of the present study is that cultural and geographic information about participants was not collected. All participants were recruited from the southwestern Virginia area, therefore the results of the present investigation should be considered in this context. In addition to including cultural and geographic information in future studies, it will be important for future work to extend these findings to diverse and underserved samples, as certain demographic variables (i.e., low SES, minority status) may be a risk for high stress (Hatch \& Dohrenwend, 2007; Myers, 2009), and thus may benefit from MBIs. Important preliminary work has demonstrated the feasibility of MBIs for diverse and underserved populations (Spears, 2019; Spears et al., 2017), and it will be important to evaluate social anhedonia in this context.

Overall, results from the current study demonstrated that MBSR directly targets social anhedonia symptoms via mediational improvements in mindfulness, whereas no such relationship was in evidence for other domains of anhedonia such as food/drink, sensory experiences, or (nonsocial) hobbies/pastimes. A potential explanation for the specificity observed here may owe to the group-administered format of MBSR. Therefore, a profitable line of future inquiry would be to experimentally examine a group versus individualized MBSR sessions, and furthermore examine potential effects of mindfulness across psychological disorders that comprise distinct social difficulties, which are perhaps themselves entrained to social anhedonic symptoms.

\section{Conclusion}

Taken together, the present study demonstrates that mindfulness is a mechanism through which anhedonic symptom improvement occurs. More specifically, these results highlight the 
specificity of anhedonic symptoms targeted by MBSR, suggesting that social anhedonia may be uniquely modified by changes in mindfulness whereas other anhedonic domains may not. Additionally, the current investigation evaluated if other theoretically-linked variables acted as mechanisms of change (i.e., stress, depression, NA) in an attempt to specify the mechanisms at play in MBSR that target anhedonic symptoms. Through this investigation, it was demonstrated that only improvements in mindfulness acted as a mediator for improvements in social anhedonia, while improvements in depression, stress, and NA did not. The results from the present study hold particular relevance for informing treatment approaches for individuals with disorders that comprise social anhedonic components. 
Author Contribution Statement: All authors were involved in the conception, drafting, and revisions of this manuscript. JAR, HST, and CNC performed the data collection for this project. Primary data analyses and interpretation of results were performed by CNC under the supervision of JAR. CNC drafted the paper, and LA, KMG, HST, and JAR provided significant feedback and revisions at all stages. All authors approved the final version of the paper for submission.

Acknowledgements: The authors would like to thank everyone who was involved in data collection including Marlene V. Strege, Gufran Mohed, Drew Weko, Grace Hinton, Jessica Feshari, Jessica Tourville and Emma Thornberg.

Funding Statement: The authors gratefully acknowledge the primary source of funding for the work outlined in this manuscript, from the Virginia Tech College of Science Dean's Discovery fund. 


\section{References}

American Psychiatric Association (2013). Diagnostic and statistical manual of mental disorders, (5th ed.) Arlington, VA: American Psychiatric Association.

Baer, R. A., Carmody, J., \& Hunsinger, M. (2012). Weekly change in mindfulness and perceived stress in a mindfulness-based stress reduction program. Journal of clinical psychology, 68(7), 755-765.

Baer, R. A., Smith, G. T., Hopkins, J., Krietemeyer, J., \& Toney, L. (2006). Five Facet Mindfulness Questionnaire. Assessment, 13, 27-45.

Baer, R. A., Smith, G. T., Lykins, E., Button, D., Krietemeyer, J., Sauer, S., ... \& Williams, J. M. G. (2008). Construct validity of the five facet mindfulness questionnaire in meditating and nonmeditating samples. Assessment, 15(3), 329-342.

Beck, A. T., Steer, R. A., \& Brown, G. (1996). Beck depression inventory-II. Psychological Assessment.

Berenbaum, H., \& Connelly, J. (1993). The effect of stress on hedonic capacity. Journal of abnormal psychology, 102(3), 474.

Bogdan, R., \& Pizzagalli, D. A. (2006). Acute stress reduces reward responsiveness: implications for depression. Biological psychiatry, 60(10), 1147-1154.

Brewer, J. A., Van Dam, N. T., \& Davis, J. H. (2015). Mindfulness and the Addictive Process: Psychological Models and Neurobiological Mechanisms. In B. Ostafin, M. Robinson, \& B. Meier (Eds.), Handbook of Mindfulness and Self-Regulation. New York, NY: Springer.

Britton, W. B., Shahar, B., Szepsenwol, O., \& Jacobs, W. J. (2012). Mindfulness-based cognitive therapy improves emotional reactivity to social stress: results from a randomized controlled trial. Behavior therapy, 43(2), 365-380. 
Carlton, C. N., Sullivan-Toole, H., Ghane, M., \& Richey, J. A. (2020a). Reward Circuitry and Motivational Deficits in Social Anxiety Disorder: What Can Be Learned From Mouse Models?. Frontiers in Neuroscience, 14.

Carlton, C. N., Sullivan-Toole, H., Strege, M. V., Ollendick, T. H., \& Richey, J. A. (2020b). Mindfulness-based interventions for adolescent social anxiety: a unique convergence of factors. Frontiers in psychology, 11, 1783.

Cernasov, P., Greene, R., Kinard, J., Halverson, T., Lowery, S., McLamb, M., ... \& Dichter, G. (2019). T78. Attenuated Default Mode Network Functional Connectivity is Associated With Improvement in Depressive Symptoms Following Mindfulness-Based Cognitive Therapy in a Transdiagnostic Anhedonic Sample. Biological Psychiatry, 85(10), S158S159.

Chapman, L. J., Chapman, J. P., \& Raulin, M. L. (1976). Scales for physical and social anhedonia. Journal of abnormal psychology, 85(4), 374.

Chi, X., Bo, A., Liu, T., Zhang, P., \& Chi, I. (2018). Effects of mindfulness-based stress reduction on depression in adolescents and young adults: a systematic review and metaanalysis. Frontiers in psychology, 9, 1034.

Clements, C. C., Zoltowski, A. R., Yankowitz, L. D., Yerys, B. E., Schultz, R. T., \& Herrington, J. D. (2018). Evaluation of the social motivation hypothesis of autism: a systematic review and meta-analysis. JAMA psychiatry, 75(8), 797-808.

Cohen, S. (1988). Perceived stress in a probability sample of the United States.

Conner, C. M., \& White, S. W. (2018). Brief report: Feasibility and preliminary efficacy of individual mindfulness therapy for adults with autism spectrum disorder. Journal of Autism and Developmental Disorders, 48(1), 290-300. 
Conner, C. M., White, S. W., Beck, K. B., Golt, J., Smith, I. C., \& Mazefsky, C. A. (2019). Improving emotion regulation ability in autism: The Emotional Awareness and Skills Enhancement (EASE) program. Autism: The International Journal of Research and Practice, 23(5), 1273-1287.

Cook, J. W., Lanza, S. T., Chu, W., Baker, T. B., \& Piper, M. E. (2017). Anhedonia: its dynamic relations with craving, negative affect, and treatment during a quit smoking attempt. Nicotine \& Tobacco Research, 19(6), 703-709.

Craske, M. G., Meuret, A. E., Ritz, T., Treanor, M., \& Dour, H. J. (2016). Treatment for anhedonia: a neuroscience driven approach. Depression and anxiety, 33(10), 927-938.

Der-Avakian, A., \& Markou, A. (2012). The neurobiology of anhedonia and other reward-related deficits. Trends in neurosciences, 35(1), 68-77.

Dichter, G. S. (2018). Motivational impairments in autism may be broader than previously thought. JAMA psychiatry, 75(8), 773-774.

Dichter, G. S., Felder, J. N., Green, S. R., Rittenberg, A. M., Sasson, N. J., \& Bodfish, J. W. (2012). Reward circuitry function in autism spectrum disorders. Social cognitive and affective neuroscience, 7(2), 160-172.

Dodell-Feder, D., \& Germine, L. (2018). Epidemiological dimensions of social anhedonia. Clinical Psychological Science, 6(5), 735-743.

Dunlop, B. W., \& Nemeroff, C. B. (2007). The role of dopamine in the pathophysiology of depression. Archives of general psychiatry, 64(3), 327-337.

Fortney, L., Luchterhand, C., Zakletskaia, L., Zgierska, A., \& Rakel, D. (2013). Abbreviated mindfulness intervention for job satisfaction, quality of life, and compassion in primary care clinicians: a pilot study. The Annals of Family Medicine, 11(5), 412-420. 
Gawrysiak, M. J., Grassetti, S. N., Greeson, J. M., Shorey, R. C., Pohlig, R., \& Baime, M. J. (2018). The many facets of mindfulness and the prediction of change following mindfulness-based stress reduction (MBSR). Journal of clinical psychology, 74(4), 523-535.

Grossman, P., Niemann, L., Schmidt, S., \& Walach, H. (2004). Mindfulness-based stress reduction and health benefits: A meta-analysis. Journal of psychosomatic research, 57(1), 35-43.

Hatch, S. L., \& Dohrenwend, B. P. (2007). Distribution of traumatic and other stressful life events by race/ethnicity, gender, SES and age: A review of the research. American journal of community psychology, 40(3-4), 313-332.

Hayes, A. F. (2012). PROCESS: A versatile computational tool for observed variable mediation, moderation, and conditional process modeling.

Höflich, A., Michenthaler, P., Kasper, S., \& Lanzenberger, R. (2019). Circuit mechanisms of reward, anhedonia, and depression. International Journal of Neuropsychopharmacology, 22(2), 105-118.

Hofmann, S. G., Sawyer, A. T., Witt, A. A., \& Oh, D. (2010). The effect of mindfulness-based therapy on anxiety and depression: A meta-analytic review. Journal of consulting and clinical psychology, 78(2), 169.

Kabat-Zinn, J. (2003). Mindfulness-based stress reduction (MBSR). Constructivism in the Human Sciences, 8(2), 73.

Kabat-Zinn, J., \& University of Massachusetts Medical Center/Worcester. Stress Reduction Clinic. (1990). Full catastrophe living: using the wisdom of your body and mind to face stress, pain, and illness. New York, N.Y.: Delacorte Press. 
Keedwell, P. A., Andrew, C., Williams, S. C., Brammer, M. J., \& Phillips, M. L. (2005). The neural correlates of anhedonia in major depressive disorder. Biological psychiatry, 58(11), 843-853.

Kenny, M. A., \& Williams, J. M. G. (2007). Treatment-resistant depressed patients show a good response to mindfulness-based cognitive therapy. Behaviour research and therapy, 45(3), $617-625$.

Khoury, B., Sharma, M., Rush, S. E., \& Fournier, C. (2015). Mindfulness-based stress reduction for healthy individuals: A meta-analysis. Journal of psychosomatic research, 78(6), 519528.

Kohls, G., Antezana, L., Mosner, M. G., Schultz, R. T., \& Yerys, B. E. (2018). Altered reward system reactivity for personalized circumscribed interests in autism. Molecular autism, $9(1), 1-12$.

Lambert, C., Da Silva, S., Ceniti, A. K., Rizvi, S. J., Foussias, G., \& Kennedy, S. H. (2018). Anhedonia in depression and schizophrenia: a transdiagnostic challenge. CNS neuroscience \& therapeutics, 24(7), 615-623.

Lee, E. H. (2012). Review of the psychometric evidence of the perceived stress scale. Asian nursing research, 6(4), 121-127.

Li, S. Y. H., \& Bressington, D. (2019). The effects of mindfulness-based stress reduction on depression, anxiety, and stress in older adults: A systematic review and metaanalysis. International Journal of Mental Health Nursing, 28(3), 635-656.

Lupien, S. J., Juster, R. P., Raymond, C., \& Marin, M. F. (2018). The effects of chronic stress on the human brain: from neurotoxicity, to vulnerability, to opportunity. Frontiers in neuroendocrinology, 49, 91-105. 
McCabe, C., Cowen, P. J., \& Harmer, C. J. (2009). Neural representation of reward in recovered depressed patients. Psychopharmacology, 205(4), 667-677.

McCabe, C., Mishor, Z., Cowen, P. J., \& Harmer, C. J. (2010). Diminished neural processing of aversive and rewarding stimuli during selective serotonin reuptake inhibitor treatment. Biological Psychiatry, 67, 439-445.

McClintock, S. M., Husain, M. M., Wisniewski, S. R., Nierenberg, A. A., Stewart, J. W., Trivedi, M. H., ... \& Rush, A. J. (2011). Residual symptoms in depressed outpatients who respond by $50 \%$ but do not remit to antidepressant medication. Journal of clinical psychopharmacology, 31(2), 180.

McMakin, D. L., Olino, T. M., Porta, G., Dietz, L. J., Emslie, G., Clarke, G., ... \& Brent, D. A. (2012). Anhedonia predicts poorer recovery among youth with selective serotonin reuptake inhibitor treatment-resistant depression. Journal of the American Academy of Child \& Adolescent Psychiatry, 51(4), 404-411.

Myers, H. F. (2009). Ethnicity-and socio-economic status-related stresses in context: an integrative review and conceptual model. Journal of behavioral medicine, 32(1), 9-19.

Morris, B. H., Bylsma, L. M., \& Rottenberg, J. (2009). Does emotion predict the course of major depressive disorder? A review of prospective studies. British Journal of Clinical Psychology, 48(3), 255-273.

Naragon-Gainey, K., Watson, D., \& Markon, K. E. (2009). Differential relations of depression and social anxiety symptoms to the facets of extraversion/positive emotionality. Journal of abnormal psychology, 118(2), 299. 
Nutt, D., Demyttenaere, K., Janka, Z., Aarre, T., Bourin, M., Canonico, P. L., ... \& Stahl, S. (2007). The other face of depression, reduced positive affect: the role of catecholamines in causation and cure. Journal of Psychopharmacology, 21(5), 461-471.

Pelizza, L., \& Ferrari, A. (2009). Anhedonia in schizophrenia and major depression: state or trait?. Annals of General Psychiatry, 8(1), 22.

Pizzagalli, D. A. (2014). Depression, stress, and anhedonia: toward a synthesis and integrated model. Annual review of clinical psychology, 10, 393-423.

Pizzagalli, D. A., Iosifescu, D., Hallett, L. A., Ratner, K. G., \& Fava, M. (2008). Reduced hedonic capacity in major depressive disorder: evidence from a probabilistic reward task. Journal of psychiatric research, 43(1), 76-87.

Pizzagalli, D. A., Jahn, A. L., \& O'Shea, J. P. (2005). Toward an objective characterization of an anhedonic phenotype: a signal-detection approach. Biological psychiatry, 57(4), 319-327.

Ribot, T. (1896). La Psychologie des Sentiment [The Psychology of Feelings]. Paris: Felix Alcan

Richey, J. A., Brewer, J. A., Sullivan-Toole, H., Strege, M. V., Kim-Spoon, J., White, S. W., \& Ollendick, T. H. (2019). Sensitivity shift theory: a developmental model of positive affect and motivational deficits in social anxiety disorder. Clinical psychology review, 72, 101756.

Rizvi, S. J., Lambert, C., \& Kennedy, S. (2018). Presentation and neurobiology of anhedonia in mood disorders: commonalities and distinctions. Current psychiatry reports, 20(2), 1-8.

Rizvi, S. J., Quilty, L. C., Sproule, B. A., Cyriac, A., Bagby, R. M., \& Kennedy, S. H. (2015). Development and validation of the Dimensional Anhedonia Rating Scale (DARS) in a community sample and individuals with major depression. Psychiatry Research, 229(1-2), 109-119. 
Rygula, R., Abumaria, N., Flügge, G., Fuchs, E., Rüther, E., \& Havemann-Reinecke, U. (2005). Anhedonia and motivational deficits in rats: impact of chronic social stress. Behavioural brain research, 162(1), 127-134.

Santorelli, S. F., Kabat-Zinn, J., Blacker, M., Meleo-Meyer, F., \& Koerbel, L. (2017). Mindfulness-based stress reduction (MBSR) authorized curriculum guide. Center for Mindfulness in Medicine, Health Care, and Society (CFM). University of Massachusetts Medical School.

Segal, Z. V., Williams, M., \& Teasdale, J. (2018). Mindfulness-based cognitive therapy for depression. Guilford Publications.

Serpa, J. G., Taylor, S. L., \& Tillisch, K. (2014). Mindfulness-based stress reduction (MBSR) reduces anxiety, depression, and suicidal ideation in veterans. Medical care, 52(12), S19S24.

Slattery, D. A., Markou, A., \& Cryan, J. F. (2007). Evaluation of reward processes in an animal model of depression. Psychopharmacology, 190(4), 555-568.

Snippe, E., Dziak, J. J., Lanza, S. T., Nyklíček, I., \& Wichers, M. (2017). The shape of change in perceived stress, negative affect, and stress sensitivity during mindfulness-based stress reduction. Mindfulness, 8(3), 728-736.

Spears, C. A. (2019). Mindfulness-based interventions for addictions among diverse and underserved populations. Current opinion in psychology, 30, 11-16.

Spears, C. A., Houchins, S. C., Bamatter, W. P., Barrueco, S., Hoover, D. S., \& Perskaudas, R. (2017). Perceptions of mindfulness in a low-income, primarily African American treatment-seeking sample. Mindfulness, 8(6), 1532-1543. 
Spijker, J., Bijl, R. V., De Graaf, R., \& Nolen, W. A. (2001). Determinants of poor 1-year outcome of DSM-III-R major depression in the general population: results of the Netherlands Mental Health Survey and Incidence Study (NEMESIS). Acta Psychiatrica Scandinavica, 103(2), 122-130.

Stanton, C. H., Holmes, A. J., Chang, S. W., \& Joormann, J. (2019). From stress to anhedonia: molecular processes through functional circuits. Trends in neurosciences, 42(1), 23-42.

Tschida, J. E., \& Yerys, B. E. (2020). A Systematic Review of the Positive Valence System in Autism Spectrum Disorder. Neuropsychology Review, 1-31.

Vrieze, E., Demyttenaere, K., Bruffaerts, R., Hermans, D., Pizzagalli, D. A., Sienaert, P., ... \& Claes, S. (2014). Dimensions in major depressive disorder and their relevance for treatment outcome. Journal of affective disorders, 155, 35-41.

Wang, Y. P., \& Gorenstein, C. (2013). Psychometric properties of the Beck Depression InventoryII: a comprehensive review. Brazilian Journal of Psychiatry, 35(4), 416-431.

Watson, D., \& Clark, L. A. (1999). The PANAS-X: Manual for the Positive and Negative Affect Schedule-Expanded Form. Retrieved from Iowa Research Online: http:/ /ir.uiowa.edu/psychology_pubs/11/

Wolf, D. H. (2006). Anhedonia in schizophrenia. Current psychiatry reports, 8(4), 322-328.

Zhou, S., Nie, L., Wang, Z., Wang, M., \& Zheng, Y. (2019). Aberrant reward dynamics in trait anticipatory anhedonia. Social cognitive and affective neuroscience, 14(8), 899-909. 


\section{Tables}

Table 1. Means and standard deviations of all study measures across all time points.

\begin{tabular}{|c|c|c|c|c|}
\hline & \multicolumn{2}{|c|}{ MBSR } & \multicolumn{2}{|c|}{ WL } \\
\hline & Mean & SD & Mean & SD \\
\hline \multicolumn{5}{|l|}{ Baseline Measures } \\
\hline FFMQ & 109.77 & 21.19 & 109.61 & 18.12 \\
\hline DARS Total & 49.00 & 9.22 & 48.61 & 11.16 \\
\hline DARS Food/Drink & 11.90 & 2.81 & 11.40 & 3.01 \\
\hline DARS Hobbies/Pastimes & 12.17 & 3.09 & 12.25 & 2.95 \\
\hline DARS Social Activities & 10.79 & 3.29 & 10.93 & 3.21 \\
\hline DARS Sensory Experiences & 14.14 & 4.55 & 14.04 & 6.20 \\
\hline PSS-10 & 22.31 & 6.09 & 21.55 & 6.79 \\
\hline BDI-II & 15.46 & 10.12 & 14.59 & 10.42 \\
\hline NA & 22.86 & 6.81 & 22.21 & 8.13 \\
\hline \multicolumn{5}{|l|}{ Midpoint Measures } \\
\hline FFMQ & 118.79 & 18.55 & 110.30 & 17.06 \\
\hline DARS Total & 48.62 & 11.83 & 48.07 & 13.44 \\
\hline DARS Food/Drink & 11.69 & 2.52 & 11.81 & 3.15 \\
\hline DARS Hobbies/Pastimes & 12.07 & 2.80 & 12.19 & 4.05 \\
\hline DARS Social Activities & 10.72 & 3.83 & 9.93 & 4.17 \\
\hline DARS Sensory Experiences & 14.14 & 4.79 & 14.15 & 5.63 \\
\hline PSS-10 & 20.38 & 6.98 & 21.07 & 6.203 \\
\hline NA & 20.47 & 5.36 & 23.80 & 7.32 \\
\hline \multicolumn{5}{|l|}{ Endpoint Measures } \\
\hline FFMQ & 127.59 & 17.06 & 114.00 & 20.25 \\
\hline DARS Total & 51.52 & 9.58 & 49.32 & 13.59 \\
\hline DARS Food/Drink & 12.34 & 2.65 & 11.14 & 4.21 \\
\hline DARS Hobbies/Pastimes & 12.66 & 2.65 & 12.79 & 4.10 \\
\hline DARS Social Activities & 12.66 & 2.54 & 9.82 & 4.39 \\
\hline DARS Sensory Experiences & 11.79 & 3.00 & 15.57 & 5.29 \\
\hline PSS-10 & 19.14 & 6.40 & 20.25 & 7.50 \\
\hline BDI-II & 9.98 & 9.18 & 16.20 & 10.96 \\
\hline NA & 20.07 & 5.92 & 21.75 & 8.12 \\
\hline
\end{tabular}


Table 2. Correlations between all study variables.

\begin{tabular}{|c|c|c|c|c|c|c|c|c|c|c|}
\hline 2. $\Delta$ Mindfulness & $.37 * *$ & - & & & & & & & & \\
\hline 4. $\triangle \mathrm{DARS}$ Food/Drink & .10 & .16 & $.67 * *$ & - & & & & & & \\
\hline 5. $\triangle \mathrm{DARS}$ Hobbies/Pastimes & -.01 & .20 & $.69 * *$ & $.40 * *$ & - & & & & & \\
\hline 6. $\triangle$ DARS Social Activities & $.35 * *$ & $.43 * *$ & $.59 * *$ & $.27 *$ & $.32 *$ & - & & & & \\
\hline 9. $\Delta \mathrm{BDI}-\mathrm{II}$ &.$- .36 * *$ & $-.45 * *$ & $-.29 *$ & $-.28 *$ & -.10 & $-.28 *$ & -.18 & $-.48 * *$ & - & \\
\hline 10. $\Delta \mathrm{NA}$ & -.14 & $-.45 * *$ & $-.32 *$ & $-.44 * *$ & -.20 & -.03 & -.20 & $-.54 * *$ & $.42 * *$ & - \\
\hline
\end{tabular}

Note: Items with $(* *)$ and bolded indicate values $p<.01$, items with $(*)$ and bolded indicate values $p<.05$. 
Table 3. Linear Regression Results.

\begin{tabular}{lccc}
\hline & \multicolumn{3}{c}{ Condition } \\
\hline & $\beta$ & $\mathrm{R}^{2}$ & $P$ \\
$\Delta$ DARS Total & .085 & .007 & .53 \\
$\Delta$ DARS Food/Drink & .095 & .009 & .48 \\
$\Delta$ DARS Hobbies/Pastimes & .009 & .000 & .95 \\
$\Delta$ DARS Social Activities & $\mathbf{. 3 4 6}$ & $\mathbf{. 1 2 0}$ & $\mathbf{. 0 1}$ \\
$\Delta$ DARS Sensory Experiences & .086 & .007 & .53 \\
$\Delta$ PSS-10 & .236 & .056 & .08 \\
$\Delta$ BDI-II & $\mathbf{. 3 6 4}$ & $\mathbf{. 1 3 3}$ & $\mathbf{. 0 1}$ \\
$\Delta$ NA & .142 & .020 & .29 \\
\hline
\end{tabular}




\section{Figures}

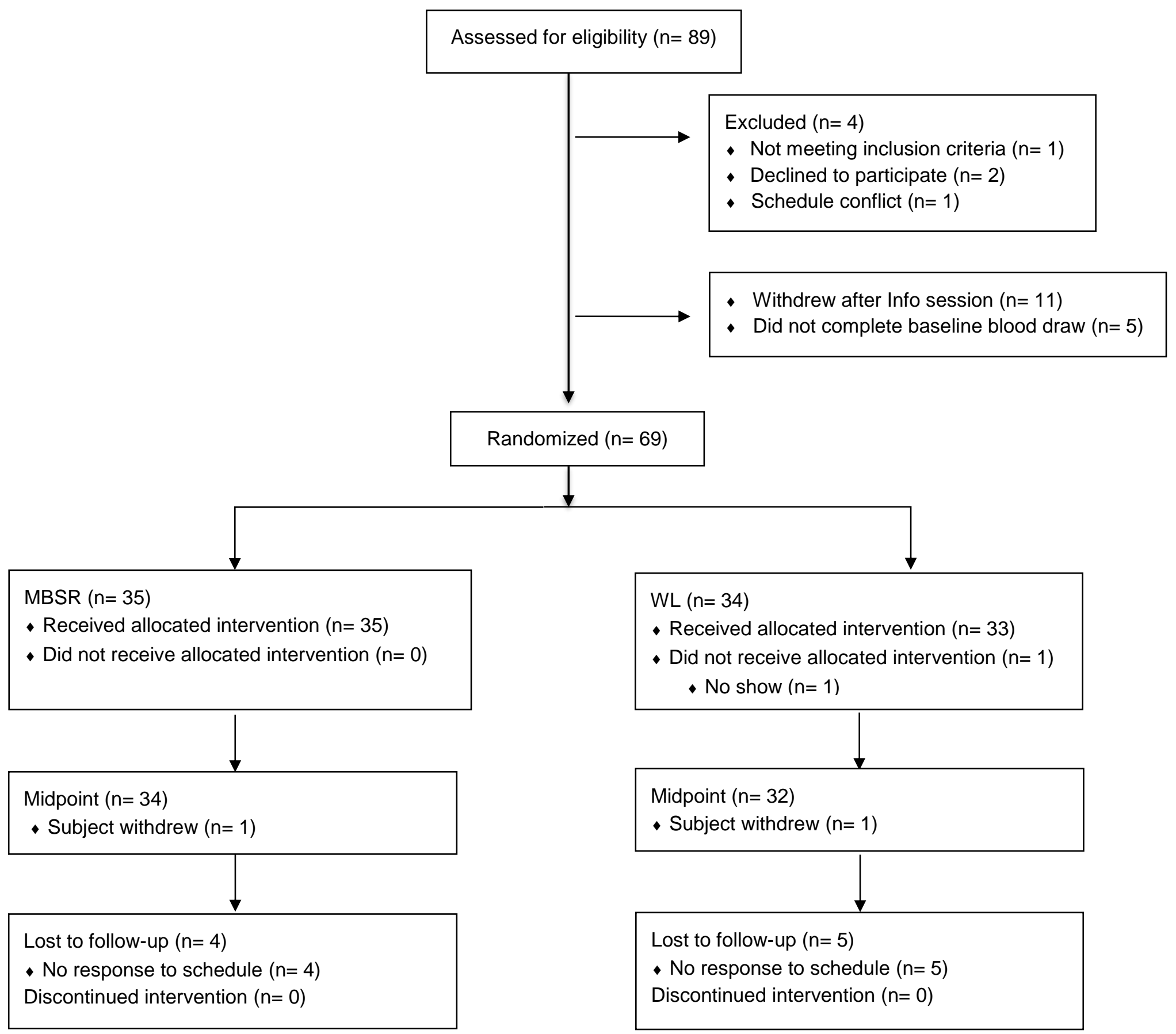

Figure 1. Consort flow chart. 


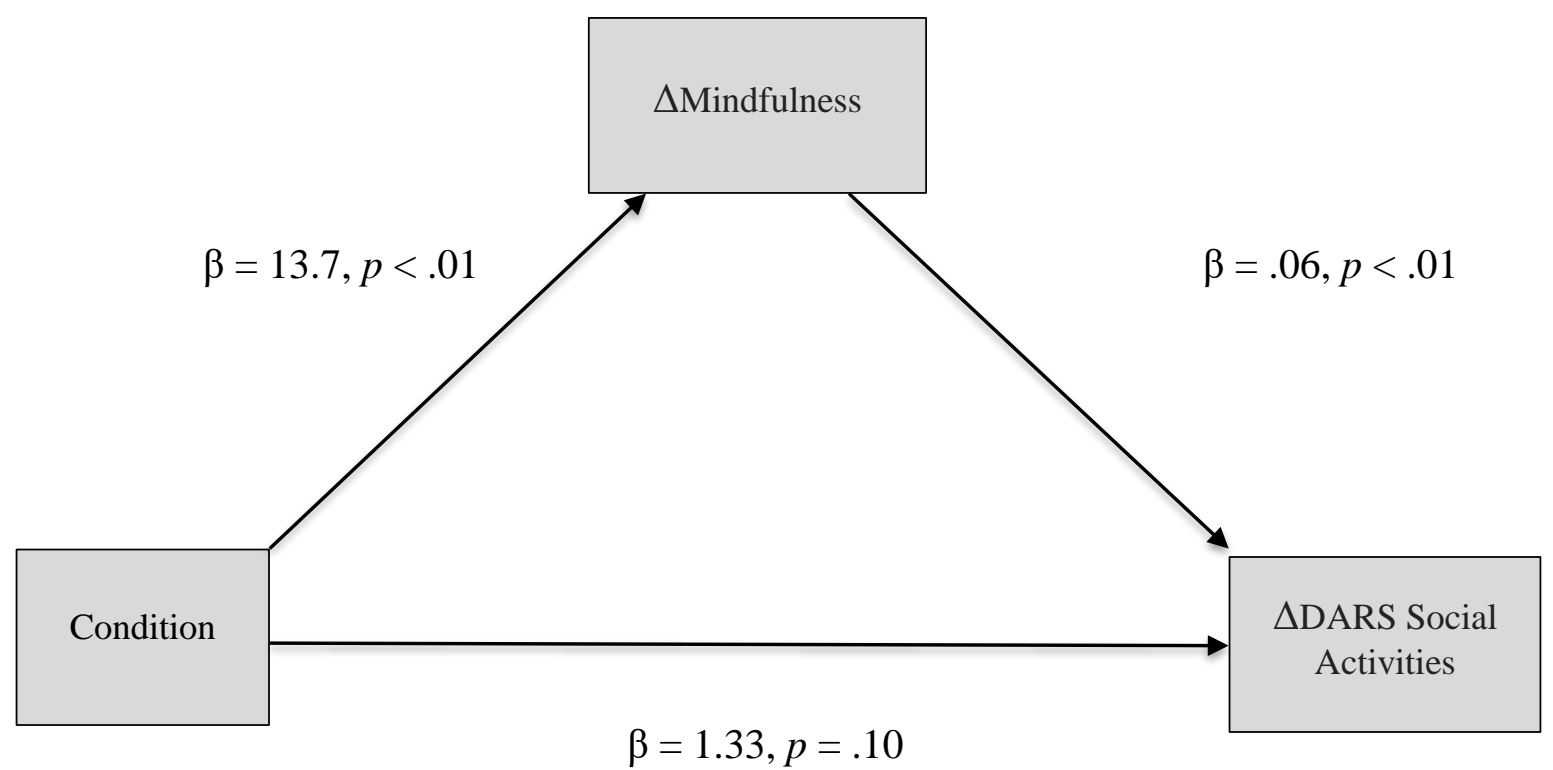

Figure 2. Model depicting the mediational role of $\Delta$ Mindfulness from baseline to endpoint on the relationship between Condition and $\triangle \mathrm{DARS}$ Social Activities. 


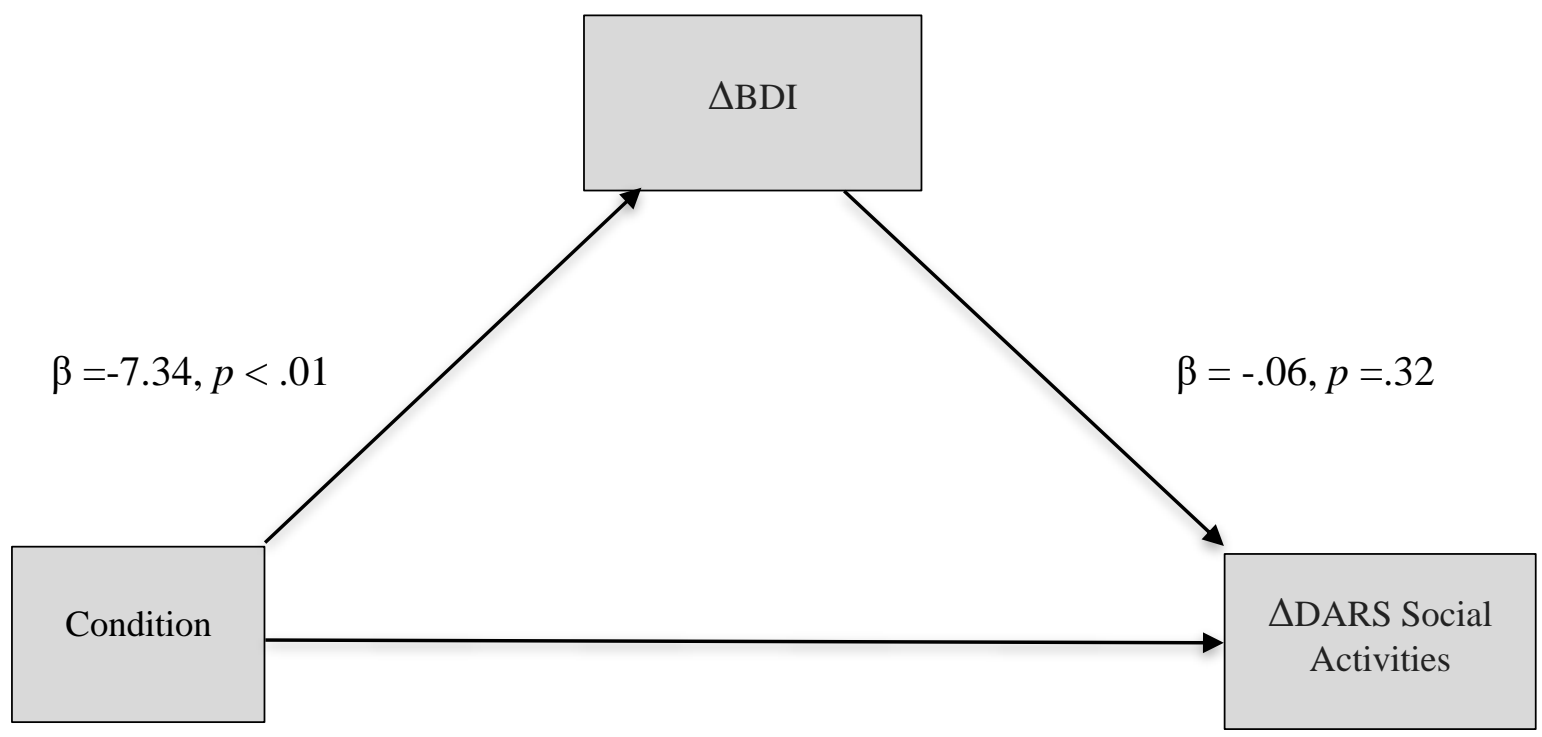

$\beta=1.78, p=.05$

Figure 3. Model depicting the mediational path of $\Delta \mathrm{BDI}-\mathrm{II}$ on the relationship between Condition and $\triangle \mathrm{DARS}$ Social Activities. 


\section{Supplementary Material}

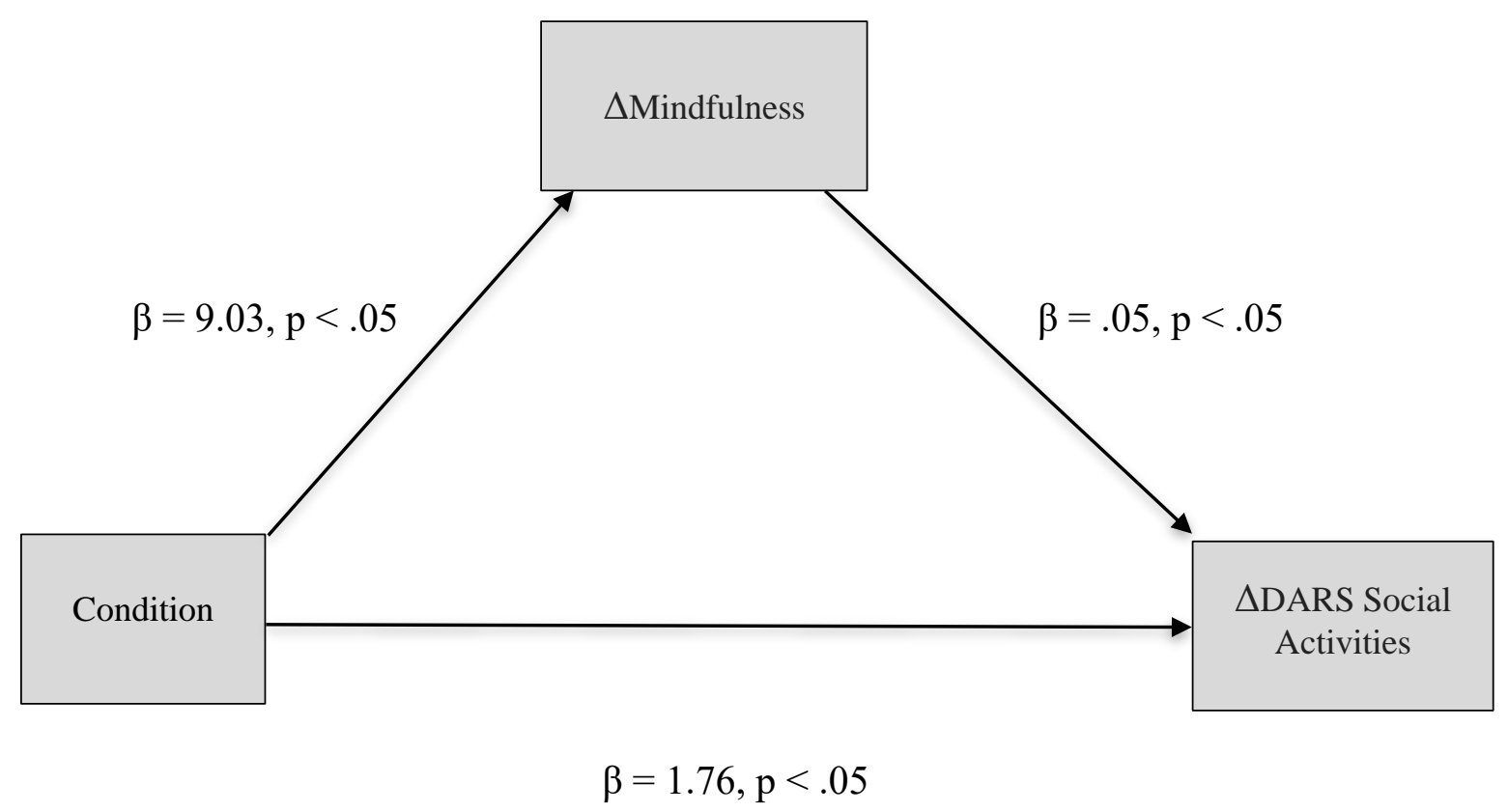

Supplemental Figure 1. Model depicting the mediational path of $\Delta$ Mindfulness from baseline to midpoint on the relationship between Condition and $\triangle$ DARS Social Activities. 